\title{
Cognitive Distortion in Suicidal Behavior
}

\author{
Honglei Yin*, Qianqian Xin, Xiang Xue and Enze Li \\ Department of Psychiatry, Nanfang Hospital, Southern Medical University, Guangzhou, China
}

*Corresponding author: Honglei Yin, Department of Psychiatry, Nanfang Hospital, Southern Medical University, Guangzhou, China

\begin{abstract}
Cognitive distortion has been proposed as one of the endophenotypes of suicidal behavior, and a therapeutic target in the suicidal patient approach. This article provides a systematic review of the existing literature testing relations between cognitive distortion and suicidality, across various diagnostic and demographic populations, using different kinds of neurocognitive tasks, together with imaging or electrophysiological test to measure the alteration of brain regions during the neurocognitive tasks. Cognitive distortion is an important factor in the generation process of suicidal behavior and should be a promising target in the prevention and treatment of suicidal behavior. Future study needs to pay attention to the consistency of samples including psychiatric diagnosis, time period between the last suicide attempt (SA) and investigation day, and the mechanisms how the genes related with suicidal behavior together with environmental factors alter the risk of suicidal behavior through their influence on cognitive distortion.
\end{abstract}

Keywords: Cognitive distortion; Suicide attempt; Suicide ideation; Imaging test

\section{Introduction}

Suicide is an important global public problem, with an estimated 800,000 people die by suicide each year worldwide and is the fifth most common cause of death and the second most common external cause of death in China [1]. Usually, suicidal behavior can be classified as suicide idea (SI), SA and suicide death. Non-fatal suicidal behaviors are more common than suicides. Although many researches discovered the gene-environmental and psychosocial characters for suicide [2-4], the risk factors of suicidal behavior remained unknown. Now, there has been great improvement in the research of the cognitive mechanisms of suicidal behavior. Cognitive distortion is not only viewed as one of the candidates endophenotypes of suicidal behavior, but also a therapeutic target in the suicidal patient approach [5]. Suicidal individuals are more likely than non-suicidal individuals to experience cognitive distortions, defined as errors in both cognitive processing and content that result in maladaptive or unhelpful interpretations of incoming stimuli [6]. different types of primary cognitive alterations are related to suicidal behavior, especially those resulting from changes in front striatal circuits [7]. Among such cognitive mechanisms can be highlighted the executive function impairment and attentional bias.

Executive function relies heavily upon three distinguishable abilities or "functions":
1) Avoiding/suppressing habitual or "pre-potent" responses (i.e., inhibition).

2) Switching between tasks or mental "sets" (i.e., shifting) and.

3) Monitoring/controlling the contents of working memory (i.e., updating) [8].

Almost all the aspects of executive function are impaired in suicidality, including poor decision making and planning, response Inhibition, verbal fluency, and so on [9]. In addition to these deficits, suicide attempters also showed a higher sensitivity to interference [10]. In the classical version of the Stroop task, the suicidal patients took significantly longer to name the colors of suicide-related words compared with other words, which means that the attentional bias exhibited by suicidal patients was highly specific [11]. Nowadays, using the modified Stroop task, together with various imaging detection methods, researchers have got further understanding of attentional bias in suicidal behavior. There is a need to synthesize evidence from cumulating literature on cognitive deficits in suicide attempters in order to get a comprehensive impression of the cognition distortion associated with a history of suicidal act. In the current paper, we systematically reviewed the literature on the cognitive distortion of suicidal behavior. 


\section{Decision making}

Many studies found that suicide attempters tended to make more risky decisions as assessed with the decision-making tasks including the Game of Dice Task (GDT), the Iowa Gambling Task (IGT) and the Cambridge Gambling Task (CGT) than control group [12-16]. A meta-analysis of 10 studies using IGT to measure decision making in patients with a history of suicidal acts in bipolar and unipolar disorder, confirmed significantly impaired decisionmaking with a moderate effect-size in bipolar disorder suicide attempters compared to unipolar and bipolar non-attempters [17]. Some studies investigated the influence of genes relevant to suicidal behavior on decision-making. found that patients with a personal history of attempted suicide carrying the specific polymorphisms of four serotonergic genes significantly improved their performance during IGT, suggesting a genetic modulation of the learning process required for advantageous decision-making [18], found that, suicide attempters with a history of sexual abuse had significantly lower IGT scores than non-sexually abused individuals, and Polymorphisms within corticotrophin releasing hormone receptor 1(CRHR1) and CRHR2 genes interacted with both childhood sexual abuse and emotional neglect to influence IGT performance [19].

There were some studies focusing on specific population groups. One study compared performance on CGT in four groups of older adults and found that the suicide attempters exhibited poorer ability to choose the likely outcome which suggesting that older suicide attempters seem to neglect outcome probability and make poor choices [20]. Most studies investigating decision making abilities in suicide attempters had used one single task and included patients with a lifetime history of suicide attempts, therefore yielded conflicting results. Some studies focused on recent suicide attempt (within $72 \mathrm{~h}$ or the last 6 months) found that, Investigation of state-dependent neuropsychological characteristics of suicidal behaviour might be essential for detecting acute suicidal crisis $[21,22]$. Functional magnetic resonance imaging (fMRI) was used to measure the brain activation following the decision making tasks in suicide attempters, one study supported a significant role for deficient risk processing, and ventral and dorsal prefrontal cortex functioning in the suicidal diathesis [23], while another suggested that a history of suicidal attempt in schizophrenia is associated with blunted brain reward activity during emotional decisionmaking [24].

\section{Response inhibition}

In 2009, a study measured two complementary aspects of response inhibition: attention-based and reward-based, found that faster reaction time to a commission error on the Immediate Memory Task (IMT) was associated with history of SA [25]. Some studies used fMRI to measure brain activation during the Go/NoGo response inhibition task, and conflict results were found. One study found decreased activity in the right anterior cingulate gyrus in SA [26], while another found no difference in brain activation [27]. Investigated the Continuous Performance Task (CPT) of 228 girls with and without attention-deficit hyperactivity disorder (ADHD), found that Childhood response inhibition could predict young-adult SI, SA, and non-suicidal self-injury [28]. Another study also found that depressed attempters were clearly distinguished by a deficit in response inhibition (Go/No-go commission errors) [29]. A recent study found that the neurocognitive assessment of response inhibition and memory recognition shows sensitivity to the recency of a SA which suggested that phasic neurocognitive deficits may serve as objective markers of short-term suicide risk [30].

\section{Working memory}

A meta-analysis in 2015 examined the association between memory deficits and vulnerability to suicidal acts in 24 studies (including 2,595 participants), four different types of memory (i.e., working memory, short- and long-term memory, and autobiographical memory) were assessed, and found that long-term memory and working memory were both more impaired in suicide attempters [31]. Several studies used MRI or fMRI to examine the structure or the functional changes in brain regions following the working memory task in patients with SI, the results were not consistent may be because of different diagnosis. One study showed that depressed patients with high suicidal ideation retained fewer negative schematic facial stimuli in white matter (WM) [32], while another study found that first-episode schizophrenia with suicidal ideation showed preserved WM integrity, and had less cognitive deficit in working memory and verbal comprehension possibly because of greater insight [33].

\section{Verbal fluency}

It is used single photon emission computed tomography (SPECT) following a verbal fluency paradigm to evaluate the perfusion of different brain regions, they found differences in regional cortical activation between the letter fluency and category fluency tasks and a blunted increase in prefrontal blood perfusion in attempted suicide patients [34]. Richard-Devantoy then reviewed seven neuropsychological tests in a total of 25 studies (2323 participants) to identify neuropsychological tests of vulnerability to suicidal acts in patients with mood disorders, and found that IGT, category verbal fluency and Stroop performance were lower in suicide attempters than in patient controls and healthy controls[5]. Several studies used near-infrared spectroscopy (NIRS), an optical neuroimaging technology that used near-infrared light to track changes in the concentration of oxygenated and deoxygenated hemoglobin ( $\mathrm{Hb})$, to measure the activation of brain regions during the verbal fluency task (VFT), and found reduced activation during VFT in several brain regions including frontal, temporal and left precentral gyrus $[35,36]$. Two studies also found prefrontal asymmetry [37] or smaller hemodynamic changes in the frontal regions [38] during VFT in depressed patients with SI.

\section{Attentional bias}

A meta-analysis of four studies, including 233 suicide attempters and 768 patient controls, showed a significant but small attentional bias toward suicide-related words in suicide attempters compared to patient controls [10]. After that time, some research used imaging or electrophysiological test together with psychological task to better understand the attentional bias in 
suicidal behavior. One study used Electroencephalographic (EEG) to record the frontal area activity during the standard and emotional Stroop tasks, found that response times to the word "suicide" were significantly slower for the high-risk group, and in the emotional Stroop task the high-risk group showed reduced activity in leftward frontal areas, suggesting limitations in the ability to regulate emotional processing via the left frontal regions [39]. Another study used the P300 component of event-related potentials (ERPs) during the spatial cueing task found that patients with low rates of suicidal behavior showed difficulty in disengaging attention from suicide-relevant stimuli [40]. Found that children with a history of SI exhibited significantly greater gaze duration toward fearful faces [41]. Investigated the emotional Stroop task (EST) of 820 college students and found that past suicide attempters were slower in responding to the word "suicide", and female past attempters showed more delayed response when their most recent attempt was made in the past 12 months [42]. Furthermore, recent research efforts are directed to assess the possible use of attention bias as a therapeutic target in patients presenting suicide behavior [43].

\section{Conclusion}

According to the overview of neurocognitive factors related to suicidal behavior, a conclusion can be yielded that cognitive distortion is an important factor in the generation process of suicidal behavior and should be a promising target in the prevention and treatment of suicidal behavior. Future research needs to pay attention to the following issues: consistency of samples including psychiatric diagnosis, time period between the last SA and investigation day and so on. Future research can focus on the mechanisms of the cognitive distortion in suicidal behavior and use the neuropsychological task to select the population with high risk of suicidal behavior. The heritability of suicide is well established. Transmission of risk appears to follow traits more than disorders like depression, hence future research can focus on the mechanisms how the genes related with suicidal behavior together with environmental factors, e.g. childhood abuse, alter the risk of suicidal behavior through their influence on cognitive distortion.

\section{Acknowledgment}

This research was funded by National Natural Science Foundation of China(NSFC) (Grant number: 81801351, PI: Honglei Yin), Guangdong Natural Science Foundation (GDNSF) (Grant number: 2017A030310530, PI: Honglei Yin).

\section{References}

1. Yin H, Xu L, Shao Y (2016) Relationship between suicide rate and economic growth and stock market in the People's Republic of China: 2004-2013. Neuropsychiatr Dis Treat 12: 3119-3128.

2. Yin H, Galfalvy H, Pantazatos SP (2016) Glucocorticoid Receptor-Related Genes: Genotype and Brain Gene Expression Relationships to Suicide and Major Depressive Disorder. Depress Anxiety 33(6): 531-540.

3. Yin H, Pantazatos, Galfalvy, Huang, Rosoklija GB, et al. (2016) A pilot integrative genomics study of GABA and glutamate neurotransmitter systems in suicide, suicidal behavior, and major depressive disorder. Am J Med Genet B Neuropsychiatr Genet 171B(3): 414-426.
4. Yin H, Galfalvy H, Zhang B (2020) Interactions of the GABRG2 polymorphisms and childhood trauma on suicide attempt and related traits in depressed patients. Journal of affective disorders 266: 447-455.

5. Richard Devantoy S, Berlim M T, Jollant F (2014) A meta-analysis of neuropsychological markers of vulnerability to suicidal behavior in mood disorders. Psychol Med 44(8): 1663-1673.

6. Jager Hyman S, Cunningham A, Wenzel A, Mattei S, Brown GK, et al. (2014) Cognitive Distortions and Suicide Attempts. Cognit Ther Res 38(4): 369-74.

7. Barredo J, Aiken E, Van 'T Wout Frank M, Greenberg BD, Carpenter LL, et al. (2019) Neuroimaging Correlates of Suicidality in Decision-Making Circuits in Posttraumatic Stress Disorder. Front Psychiatry 10: 44.

8. Bredemeier K, Miller I W (2005) Executive function and suicidality: A systematic qualitative review. Clin Psychol Rev 40: 170-183.

9. Legris J, Van Reekum R (2006) The neuropsychological correlates of borderline personality disorder and suicidal behaviour. Can J Psychiatry 51(3): 131-142.

10. Richard Devantoy S, Ding Y, Turecki G (2016) Attentional bias toward suicide-relevant information in suicide attempters: A cross-sectional study and a meta-analysis. J Affect Disord 196: 101-108.

11. Becker ES, Strohbach D, Rinck M (1999) A specific attentional bias in suicide attempters. The J Nerv Ment Dis 187(12): 730-735.

12. Jollant $F$, Bellivier F, Leboyer $M$, Astruc $B$, Torres $S$, et al. (2005) Impaired decision making in suicide attempters. Am J Psychiatry 162(2): 304-310.

13. Jollant F, Guillaume S, Jaussent I, Castelnau D, Malafosse A, et al. (2007) Impaired decision-making in suicide attempters may increase the risk of problems in affective relationships. J Affect Disord 99(1-3): 59-62.

14. Bridge JA, Mcbee-Strayer SM, Cannon EA, Sheftall AH, Reynolds B, et al. (2012) Impaired decision making in adolescent suicide attempters. J Am Acad Child and Adolesc Psychiatry 51(4): 394-403.

15. Hoehne A, Richard-Devantoy S, Ding Y (2015) First-degree relatives of suicide completers may have impaired decision-making but functional cognitive control. J psychiatr Res 68: 192-197.

16. Ackerman JP, Mcbee-Strayer SM, Mendoza K, Stevens J, Sheftall AH, et al. (2015) Risk-sensitive decision-making deficit in adolescent suicide attempters. J child Adolesc psychopharmacol 25(2): 109-113.

17. Richard Devantoy S, Olie E, Guillaume S (2016) Decision-making in unipolar or bipolar suicide attempters. J Affective Disord 190: 128-136.

18. Jollant F, Buresi C, Guillaume S, Jaussent I, Bellivier F, et al. (2007) The influence of four serotonin-related genes on decision-making in suicide attempters. Am J Med Genet B Neuropsychiatr Genet. 144B(5): 615-624.

19. Guillaume S, Perroud N, Jollant F, Jaussent I, Olié E, et al. (2013) HPA axis genes may modulate the effect of childhood adversities on decisionmaking in suicide attempters. J Psychiatr Res 47(2): 259-265.

20. Clark L, Dombrovski AY, Siegle G J, Butters MA, Shollenberger CL, et al. (2011) Impairment in risk-sensitive decision-making in older suicide attempters with depression. Psychol Aging 26(2): 321-330.

21. Deisenhammer EA, Schmid SK, Kemmler G (2018) Decision making under risk and under ambiguity in depressed suicide attempters, depressed non-attempters and healthy controls. J Affect Disord 226: 261-266.

22. Hegedus KM, Szkaliczki A, Gal BI, Andó B, Janka Z, et al. (2018) Decisionmaking performance of depressed patients within $72 \mathrm{~h}$ following a suicide attempt. J Affect Disord 235: 583-588.

23. Ding Y, Pereira F, Hoehne A, Beaulieu MM, Lepage M, et al. (2017) Altered brain processing of decision-making in healthy first-degree biological relatives of suicide completers. Mol Psychiatry 22(8): 1149-1154. 
24. Potvin S, Tikasz A, Richard Devantoy S (2018) History of Suicide Attempt Is Associated with Reduced Medial Prefrontal Cortex Activity during Emotional Decision-Making among Men with Schizophrenia: An Exploratory fMRI Study. Schizophrenia research and treatment pp. 9898654.

25. Swann A C, Lijffijt M, Lane S D (2009) Severity of bipolar disorder is associated with impairment of response inhibition. J Affect Disord 116(1-2): 30-36.

26. Pan L A, Batezati Alves S C, Almeida J R, Segreti A, Akkal D, et al. (2011) Dissociable patterns of neural activity during response inhibition in depressed adolescents with and without suicidal behavior. J Am Acad Child Adolesc Psychiatry 50(6): 602-611 e3.

27. Richard Devantoy S, Ding Y, Lepage M (2016) Cognitive inhibition in depression and suicidal behavior: a neuroimaging study. Psychol Med 46(5): 933-944.

28. Meza JI, Owens EB, Hinshaw SP (2016) Response Inhibition, Peer Preference and Victimization, and Self-Harm: Longitudinal Associations in Young Adult Women with and without ADHD. J Abnorm Child Psychol 44(2): 323-334.

29. Moniz M, De Jesus S N, Pacheco A (2017) The Influence of Planning and Response Inhibition on Cognitive Functioning of Non-Psychotic Unipolar Depressed Suicide Attempters. Europe's journal of psychology 13(4): 717-732.

30. Interian A, Myers C E, Chesin M S (2019) Towards the objective assessment of suicidal states: Some neurocognitive deficits may be temporally related to suicide attempt. Psychiatry research pp. 112624.

31. Richard-Devantoy S, Berlim M T, Jollant F (2015) Suicidal behaviour and memory: A systematic review and meta-analysis. World J Biol Psychiatry 16(8): 544-566.

32. Xie W, Li H, Zou Y (2018) A suicidal mind tends to maintain less negative information in visual working memory. Psychiatr Res 262: 549-557.

33. Long Y, Ouyang X, Liu Z, Chen X, Hu X, et al. (2018) Associations Among Suicidal Ideation, White Matter Integrity and Cognitive Deficit in FirstEpisode Schizophrenia. Front Psychiatry 9: 300-391.

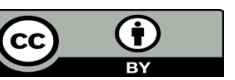

This work is licensed under Creative

Commons Attribution 4.0 License

To Submit Your Article Click Here:

Submit Article
34. Audenaert K, Goethals I, Van Laere K, Lahorte P, Brans B, et al. (2002) SPECT neuropsychological activation procedure with the Verbal Fluency Test in attempted suicide patients. Nucl Med Commun 23(9): 907-916.

35. Tsujii N, Mikawa W, Tsujimoto E, Adachi T, Niwa A, et al. (2017) Reduced left precentral regional responses in patients with major depressive disorder and history of suicide attempts. PLoS one 12(4): e0175249.

36. Hirose T, Tsujii N, Mikawa W (2018) Delayed hemodynamic responses associated with a history of suicide attempts in bipolar disorder: a multichannel near-infrared spectroscopy study. Psychiatry Res Neuroimaging 280: 15-21.

37. Baik S Y, Kim J Y, Choi J, Baek JY, Park Y, et al. (2019) Prefrontal Asymmetry during Cognitive Tasks and its Relationship with Suicide Ideation in Major Depressive Disorder: An fNIRS Study. Diagnostics (Basel) 9(4): E193.

38. Pu S, Nakagome K, Yamada T, et al. (2015) Suicidal ideation is associated with reduced prefrontal activation during a verbal fluency task in patients with major depressive disorder. J Affect Disord 181: 9-17.

39. Thompson C, Ong ELC (2018) The Association Between Suicidal Behavior, Attentional Control, and Frontal Asymmetry. Front Psychiatry 9: 70-79.

40. Baik S Y, Jeong M, Kim H S (2018) ERP investigation of attentional disengagement from suicide-relevant information in patients with major depressive disorder. J Affect Disord 225: 357-364.

41. Tsypes A, Owens M, Gibb BE (2017) Suicidal ideation and attentional biases in children: An eye-tracking study. J Affect Disord 222: 133-137.

42. Chung Y, Jeglic EL (2016) Use of the Modified Emotional Stroop Task to Detect Suicidality in College Population. Suicide Life Threat Behav 46(1): 55-66.

43. Da Silva A G, Malloy Diniz L F, Garcia M S, Figueiredo CGS, Figueiredo RN, et al. (2018) Cognition As a Therapeutic Target in the Suicidal Patient Approach. Front Psychiatry 9: 1-31.

DOI: $10.32474 /$ SJPBS.2020.03.000168

\begin{tabular}{|l|l|}
\hline SJPBS & $\begin{array}{c}\text { Scholarly Journal of Psychology } \\
\text { and Behavioral Sciences }\end{array}$ \\
Assets of Publishing with us
\end{tabular}

